\title{
Intravitreal Aflibercept versus Laser Photocoagulation in Asian Patients with Diabetic Macular Edema: The VIVID-East Study
}

This article was published in the following Dove Press journal: Clinical Ophthalmology

\author{
You-Xin Chen,' Xiao-Xin \\ $\mathrm{Li}^{2}$ Young Hee Yoon, ${ }^{3}$ \\ Xiaodong Sun, ${ }^{4}$ Yury \\ Astakhov, ${ }^{5}$ Gezhi Xu, ${ }^{6}$ Hui \\ Wang, ${ }^{7}$ Xiaowei Ren, ${ }^{8}$ \\ Friedrich Asmus ${ }^{9}$

\section{On behalf of the VIVID-East investigators}

\begin{abstract}
'Department of Ophthalmology, Peking Union Medical College, Beijing, People's Republic of China; ${ }^{2}$ Ophthalmology Department, People's Eye Center of Peking University People's Hospital, Beijing, People's Republic of China; ${ }^{3}$ Department of Ophthalmology, Asan Medical Center, University of Ulsan College of Medicine, Seoul, Republic of Korea; ${ }^{4}$ Department of Ophthalmology, Shanghai Jiao Tong University School of Medicine, Shanghai, People's Republic of China; ${ }^{5}$ Department of Ophthalmology, Pavlov First St. Petersburg State Medical University, St. Petersburg, Russia; ${ }^{6}$ Department of Ophthalmology and Vision Sciences, Eye and ENT Hospital, Shanghai Medical College, Fudan University, Shanghai, People's Republic of China; ${ }^{7}$ Clinical Development, Ophthalmology, Bayer Healthcare Inc Shanghai, People's Republic of China; ${ }^{8}$ Statistics and Data Insights, Bayer Healthcare Inc, Beijing, People's Republic of China; ${ }^{9}$ University Hospital of Tübingen, Tübingen, Germany
\end{abstract}

Correspondence: Friedrich Asmus Center for Neurology, University Hospital of Tübingen, Hoppe-Seyler Strasse 3, Tübingen, Germany Tel +49 I797463010

Email friedrich.asmus@gmail.com
Purpose: The prevalence of diabetes and diabetes-related complications, including diabetic macular edema (DME), is increasing in Asia and worldwide.

Methods: VIVID-East was a 12-month, double-masked, randomized, active-controlled, Phase 3 trial (NCT01783886) enrolling adult patients (aged $\geq 18$ years) with DME at 25 centers across China, Hong Kong, Republic of Korea, and Russia. Eyes were randomized 1:1:1 to $2 \mathrm{mg}$ intravitreal aflibercept (IVT-AFL) every 4 weeks ( $2 \mathrm{q} 4 ; \mathrm{N}=127), 2 \mathrm{mg}$ IVTAFL every 8 weeks (after 5 initial monthly doses from baseline to week 16) with sham injections on nontreatment visits $(2 \mathrm{q} 8 ; \mathrm{N}=127)$, or macular laser photocoagulation at baseline and sham injections at every visit (laser control group; $\mathrm{N}=124$ ). The primary efficacy endpoint was mean change in best corrected visual acuity (BCVA) from baseline to week 52 . Results: Compared with baseline, at week 52 the mean (SE) BCVA in the $2 \mathrm{q} 4$ and $2 \mathrm{q} 8$ groups gained $+13.6(0.9)$ and +13.1 (1.0) letters, respectively, versus -0.5 (1.4) letters in the laser group $(\mathrm{P}<0.0001$ for both). A significantly higher proportion of patients treated with IVT-AFL ( $2 \mathrm{q} 4$ and $2 \mathrm{q} 8$ ) achieved $\mathrm{a} \geq 10$-letter or $\geq 15$-letter gain compared with laser (both $\mathrm{P}<0.0001)$ ( $\geq 10$-letter gain: $70.9 \%, 62.7 \%$, and $23.4 \%$, respectively; $\geq 15$-letter gain: $43.3 \%, 36.5 \%$, and $12.1 \%$, respectively). Mean reduction in central retinal thickness from baseline to week 52 was significantly greater with IVT-AFL versus laser treatment. Incidence of treatment-emergent adverse events was low and similar across groups; conjunctival hemorrhage (11.8\%), retinal hemorrhage $(8.7 \%)$, retinal aneurysm $(7.5 \%)$, and retinal exudates $(5.5 \%)$ being the most frequently reported. Visual and anatomic outcomes in the Chinese subgroup were consistent with the overall population.

Conclusion: IVT-AFL treatment resulted in significant visual and anatomic improvements in Asian patients with DME. Treatment benefits observed in the overall study population were mirrored in the subgroup of Chinese patients, who made up the largest population group in the study.

Study Registration: NCT01783886.

Keywords: Asia, Chinese, intravitreal aflibercept, diabetic macular edema

\section{Introduction}

One-third of patients with diabetes have ocular complications. ${ }^{1}$ Retinal vascular leakage can lead to an accumulation of excess extracellular fluid, manifesting as diabetic macular edema (DME). ${ }^{2}$ Half of all people living with diabetes mellitus reside in Asia, ${ }^{3}$ and the prevalence of diabetes mellitus in China increased 14-fold between 1980 and $2008 .^{3}$ The prevalence of diabetes and diabetes-related complications, including DME, is increasing worldwide, ${ }^{1,3}$ and is reported to be 5.2 $11.2 \%$ of all cases of diabetes in China. ${ }^{3}$ 
The role of vascular endothelial growth factor (VEGF) in the pathophysiology of diabetic retinopathy and DME has been well documented. ${ }^{2,4}$ VEGF modulates the development and maintenance of the vasculature, and its expression is upregulated in patients with diabetes, resulting in excessive angiogenesis and increased vascular permeability, including that in the eyes. ${ }^{2}$ Intravitreal antiVEGF therapy has become the standard of care for managing DME due to the favorable benefit-risk profile. ${ }^{5}$ However, it has been suggested that DME diagnosis and management remain suboptimal in many patients with diabetes, particularly in Asia where limited screening is conducted and patients tend to present far later in the course of their DME than in other regions. ${ }^{5}$

The phase 3 VIVID and VISTA studies compared visual and anatomic outcomes in patients with DME treated with intravitreal aflibercept (IVT-AFL) every 4 weeks or every 8 weeks (after 5 initial monthly doses) versus those treated with laser photocoagulation at 127 sites in the United States, Europe, Japan, and Australia. ${ }^{6-8}$ Findings showed that IVT-AFL was superior to laser treatment in improving functional and anatomic outcomes at 52 weeks. ${ }^{6}$ The treatment benefits were maintained over 148 weeks, providing evidence for a therapeutic option with a longer injection interval, which reduces the treatment burden on patients and physicians. ${ }^{8}$ To date, information regarding the efficacy and safety of intravitreal anti-VEGF therapy in Asian patients has been limited. The present 52week study, VIVID-East, evaluated the efficacy and safety of IVT-AFL either every 4 weeks or every 8 weeks (after 5 initial monthly doses) compared with macular laser photocoagulation (with sham injections) in Asian patients with DME, including a subgroup of patients from China.

\section{Methods}

\section{Study Design}

VIVID-East (NCT01783886) was a 12-month doublemasked, randomized, active-controlled, phase 3 trial conducted in patients with DME. The study was carried out at 25 centers across China, Hong Kong, the Republic of Korea, and Russia (see supplementary Information). Each clinical site's respective Institutional Review Board reviewed and approved the protocol, and the study was conducted in accordance with the ethical principles in the Declaration of Helsinki and the International Conference on Harmonization E6 Good Clinical Practice Guideline.
All participants provided written informed consent prior to enrollment.

\section{Participants and Treatments}

Adult patients (aged $\geq 18$ years) with type 1 or 2 diabetes mellitus who presented with clinically significant DME involving the center of the macula (defined as the area of the center subfield of optical coherence tomography [OCT] $)$ in the study eye were included. Eligible patients had central retinal thickness (CRT), as assessed by OCT, $\geq 300 \mu \mathrm{m}$ and the best corrected visual acuity (BCVA) Early Treatment Diabetic Retinopathy Study (ETDRS) letter score between 73 and 24 (20/40 to 20/320 Snellen equivalent) in the study eye. Only 1 eye per patient was enrolled in the study. Patients were excluded from the study if they had an ocular condition with a poorer prognosis in the fellow eye than in the study eye, any surgical interventions or laser photocoagulation in the study eye within 120 and 90 days of day 1, respectively, any treatments with corticosteroids or anti-angiogenic drugs in either eye within 90 days of day 1, active proliferative diabetic retinopathy in the study eye, or a history of idiopathic or autoimmune uveitis in the study eye.

Eyes were randomized 1:1:1 to receive either: $2 \mathrm{mg}$ IVT-AFL every 4 weeks (2q4; a maximum of 13 injections) with sham laser; $2 \mathrm{mg}$ IVT-AFL every 8 weeks (after 5 initial monthly doses from baseline to week 16; a maximum of 9 injections) with sham injections on nontreatment visits $(2 q 8)$ with sham laser, or macular laser photocoagulation using the modified ETDRS protocol at baseline and sham IVT-AFL injections at every visit (laser control group). All participants were evaluated for laser retreatment at week 12. Criteria for laser treatment were thickening of the retina at or within $500 \mu \mathrm{m}$ of the center of the macula; hard exudates at or within $500 \mu \mathrm{m}$ of the center of the macula, if associated with thickening of adjacent retina; or a zone or zones of retinal thickening 1 disc area or larger, any part of which is within 1 disc diameter of the center of the macula. In participants meeting the criteria for laser treatment, the $2 q 4$ and $2 q 8$ groups received sham laser and those in the laser group received active laser, but not more frequently than every 12 weeks.

Study eyes in all treatment groups were reevaluated for treatment at week 24 and could receive additional (rescue) treatment from week 24 onward if they lost, owing to worsening $\mathrm{DME}, \geq 10$ letters at 2 consecutive visits or $\geq 15$ letters from the best previous evaluation, and BCVA became worse than that at baseline. When criteria for 
additional treatment were met, study eyes in the $2 \mathrm{q} 4$ and 2q8 groups received active laser treatment (rather than sham), but not more often than once every 12 weeks, while patients in the laser group received 5 initial monthly doses of $2 \mathrm{mg}$ IVT-AFL followed by dosing every 8 weeks.

\section{Outcome Measures}

The primary efficacy endpoint was the mean change in BCVA in ETDRS letter score from baseline to week 52. The secondary efficacy endpoints were (a) proportion of eyes that gained $\geq 10$ ETDRS letters from baseline to week 52 ; (b) proportion of eyes that gained $\geq 15$ ETDRS letters from baseline to week 52; (c) proportion of eyes with a $\geq 2$-step improvement from baseline in the Diabetic Retinopathy Severity Scale (DRSS), as assessed by fundus photography from baseline to week 52; (d) change in CRT from baseline to week 52 as assessed on OCT; (e) change from baseline to week 52 in National Eye Institute Visual Functioning Questionnaire-25 (NEI VFQ-25) near activities subscales; and ( $\mathrm{f}$ ) change from baseline to week 52 in NEI VFQ-25 distance activities subscales. According to the NEI VFQ-25, near activities are defined as reading ordinary print in newspapers, performing work or hobbies requiring near vision, or finding something on a crowded shelf. Distance activities are defined as reading street signs or names on stores, and going downstairs, steps, or curbs. Safety outcomes were also assessed.

\section{Statistical Analyses}

Demographic data and baseline characteristics were summarized using descriptive statistics. The primary efficacy endpoint was evaluated for the full analysis set (FAS) population, which comprised all randomized participants who received any study treatment, had a baseline measurement of BCVA, and had $\geq 1$ postbaseline assessment of BCVA. The primary endpoint was assessed using an analysis of covariance model with baseline BCVA measurement as a covariate and treatment group and geographic region (China vs Russia vs Hong Kong/Republic of Korea) as fixed factors.

Analyses for all secondary efficacy endpoints were conducted in the FAS population and were tested for superiority of either IVT-AFL group over the laser group in a hierarchical testing procedure following order (a) to (f), as described in the "Outcome measures" section. A P-value $<0.05$ was considered to represent superiority for a given endpoint in the hierarchical analysis of secondary endpoints only if all preceding endpoint comparisons in the hierarchy (including the primary endpoint) were shown to be statistically significant at $\mathrm{P}<0.025$. The proportion of patients who gained $\geq 10$ / $\geq 15$ ETDRS letters from baseline to week 52 was compared using a Cochran-Mantel-Haenszel test, stratifying by geographic region. Last observation carried forward was the primary method used to replace missing values. Leastsquares mean values are reported. For patients receiving additional (rescue) treatment, all efficacy data were censored from the time rescue treatment was initiated, and the last observation before rescue treatment was given was carried forward.

\section{Results}

A total of 381 patients were randomized across the three study arms (Figure 1). Three patients randomized to the laser group were withdrawn from the study prior to receiving treatment due to study protocol deviations $(n=2)$ and withdrawal of consent $(n=1)$. A total of 355 patients (93.2\%) completed the 52-week study (Figure 1). Baseline demographics and characteristics were well balanced across treatment arms (Table 1). Mean treatment compliance was at least $99 \%$ in all dose groups through week 52. A total of $91.9 \%$ (in the laser photocoagulation group) to $93.7 \%$ (in the $2 \mathrm{q} 8$ group) of patients received all scheduled injections (active or sham). The mean (SD) number of treatments was 12.6 (1.9) and 8.7 (1.1) IVT-AFL injections in the $2 q 4$ and the $2 q 8$ groups, respectively, and 2.4 (1.1) laser treatments in the laser photocoagulation group. One $(0.8 \%)$ and 4 (3.1\%) patients in the $2 q 4$ and $2 q 8$ groups, respectively, received 1 active laser treatment from week 24 onward, while 8 patients $(6.3 \%)$ in the $2 q 4$ group and $4(3.1 \%)$ in the $2 \mathrm{q} 8$ group met additional treatment criteria but did not receive laser treatment. One patient in the $2 \mathrm{q} 8$ group received additional laser treatment without having met the additional treatment criteria. Forty-five patients $(36.3 \%)$ in the laser photocoagulation group received additional treatments with IVT-AFL. The mean (SD) time to first additional IVT-AFL treatment from week 24 onward was 48.7 (56.5) days, and the mean (SD) number of additional treatments received was 4.6 (1.6) with a range of 2-7 treatments.

\section{Efficacy}

IVT-AFL was associated with a clinically meaningful and statistically significant improvement from baseline in BCVA compared with laser photocoagulation at 52 weeks. The mean (SE) BCVA in the $2 \mathrm{q} 4$ and $2 \mathrm{q} 8$ groups was +13.6 (0.9) and +13.1 (1.0) letters, respectively, compared with 


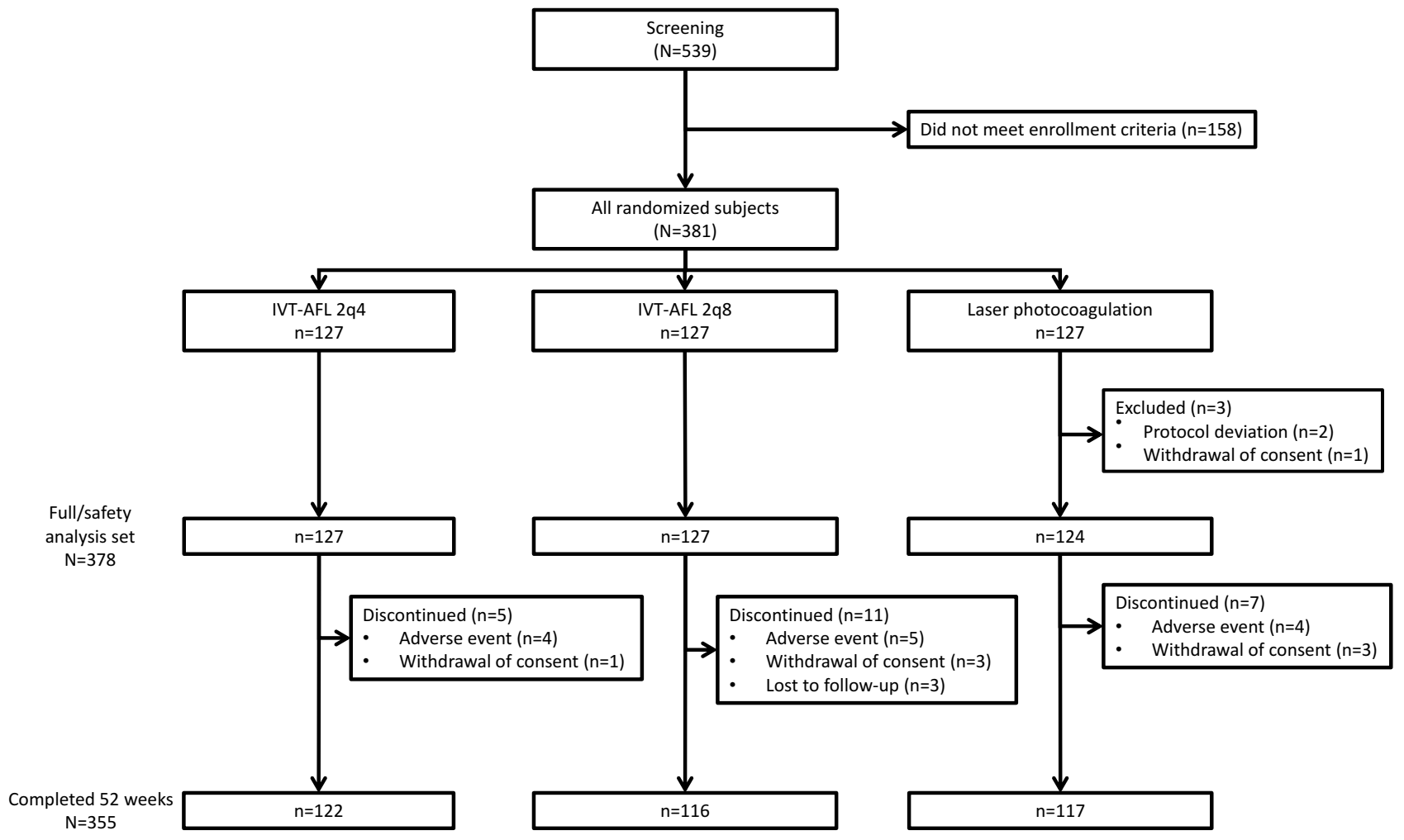

Figure I Consolidated standards of reporting trial (CONSORT) diagram of study participant disposition.

Abbreviations: 2q4, 2 mg every 4 weeks; 2q8, 2 mg every 8 weeks; IVT-AFL, intravitreal aflibercept.

-0.5 (1.4) letters in the laser photocoagulation group (both $\mathrm{P}<0.0001$ ) (Figure 2A). An improvement in BCVA versus baseline was observed as early as week 4 with IVT-AFL $2 q 4$ and $2 \mathrm{q} 8$, with continued improvement observed through week 52 in both IVT-AFL treatment groups. BCVA improved in patients randomized to laser photocoagulation from baseline through week 12 , after which a gradual decline was observed through to week 52 .

At week 52 , the proportion of patients that gained $\geq 10$ letters from baseline in the IVT-AFL $2 q 4$ and $2 q 8$ groups compared with the laser group was $70.9 \%$ and $62.7 \%$, respectively, versus $23.4 \%$, (both $\mathrm{P}<0.0001$ ). A significantly higher proportion of patients treated with IVT-AFL ( $2 q 4$ and $2 q 8$ ) also achieved a $\geq 15$-letter gain in ETDRS letters compared with laser photocoagulation (both $\mathrm{P}<0.0001$ ) (Figure 3A). Similarly, significantly greater proportions of patients treated with IVT-AFL $2 \mathrm{q} 4$ and $2 \mathrm{q} 8$ compared with those treated with laser photocoagulation had a $\geq 2$-step improvement in DRSS score from baseline to week $52(60.7 \%$ and $62.3 \%$, respectively, versus $21.7 \%$; both $\mathrm{P}<0.0001$ ) (Figure $3 \mathrm{~B}$ ). The mean change in CRT from baseline to week 52 was significantly greater in both IVT-AFL $2 q 4$ and $2 q 8$ groups compared with the laser group $(-231.1 \mu \mathrm{m}$ and $-232.0 \mu \mathrm{m}$, respectively, versus $-100.6 \mu \mathrm{m}$; both $\mathrm{P}<0.0001)$.

From baseline to week 52, the mean (SE) change in NEI VFQ-25 scores for near activities was significantly greater in both IVT-AFL $2 \mathrm{q} 4$ and $2 \mathrm{q} 8$ groups compared with the laser group (5.16 [2.30] and 6.25 [2.23], respectively, versus -0.62 [2.38]; both $\mathrm{P}<0.05)$. For distance activities, the mean (SE) change from baseline to week 52 was 3.36 (2.09) and 8.55 (1.90) for the $2 \mathrm{q} 4$ and $2 \mathrm{q} 8$ groups, respectively, versus 0.54 (2.17) for the laser group $(\mathrm{P}<0.001$ for the $2 \mathrm{q} 8$ group).

\section{Chinese Subgroup}

The largest ethnic subgroup randomized in VIVID-East was comprised of Chinese patients $(n=301 ; 79.6 \%$ of the overall study population). Of these, 280 (92.4\%) patients completed the 52-week follow-up period. Baseline demographics and disease characteristics were comparable to the overall study population (Table 1). The proportion of the Chinese subgroup patient population who received all planned injections during the treatment period was $91.1 \%$ and $94.1 \%$ in the $2 \mathrm{q} 4$ and $2 \mathrm{q} 8$ groups, respectively. 
Table I Baseline Demographics and Disease Characteristics

\begin{tabular}{|c|c|c|c|}
\hline Characteristic & IVT-AFL 2q4 (N=|27) & IVT-AFL 2q8 (N=|27) & Laser Photocoagulation $(\mathrm{N}=124)$ \\
\hline \multicolumn{4}{|l|}{ Total population } \\
\hline Female, n (\%) & $68(53.5)$ & $60(47.2)$ & $60(48.4)$ \\
\hline Mean age, years (SD) & $59.3(10.3)$ & $57.6(10.1)$ & $58.8(10.5)$ \\
\hline \multicolumn{4}{|l|}{ Race, n (\%) } \\
\hline White & $10(7.9)$ & $10(7.9)$ & $9(7.3)$ \\
\hline Asian & $117(92.1)$ & $117(92.1)$ & $115(92.7)$ \\
\hline \multicolumn{4}{|l|}{ Geographic region, n (\%) } \\
\hline China & $101(79.5)$ & $101(79.5)$ & $99(79.8)$ \\
\hline Russia & $10(7.9)$ & $10(7.9)$ & $9(7.3)$ \\
\hline Hong Kong/Republic of Korea & $16(12.6)$ & $16(12.6)$ & $16(12.9)$ \\
\hline Mean $\mathrm{HbA}_{\mathrm{Ic}}, \%(\mathrm{SD})^{\mathrm{a}}$ & $7.6(1.4)$ & $7.3(1.3)$ & $7.3(1.4)$ \\
\hline Patients with $\mathrm{HbA}_{\mathrm{Ic}}>8 \%, \mathrm{n}(\%)$ & $41(32.3)$ & $34(26.8)$ & $33(26.6)$ \\
\hline Mean duration of diabetes, years $(\mathrm{SD})^{\mathrm{b}}$ & $12.9(7.7)$ & $11.5(7.9)$ & $12.6(7.8)$ \\
\hline Mean BCVA, letters (SD) & $55.6(12.1)$ & $57.1(12.5)$ & $55.1(14.2)$ \\
\hline Mean CRT, $\mu \mathrm{m}(\mathrm{SD})$ & $526.3(164.4)$ & $520.3(154.8)$ & $527.7(170.5)$ \\
\hline \multicolumn{4}{|l|}{ ETDRS DRSS at baseline, $\mathrm{n}(\%)$} \\
\hline 35 & $4(3.1)$ & $4(3.1)$ & $6(4.8)$ \\
\hline 43 & $17(13.4)$ & $21(16.5)$ & $8(6.5)$ \\
\hline 47 & $27(21.3)$ & $19(15.0)$ & $24(19.4)$ \\
\hline 53 & $76(59.8)$ & $77(60.6)$ & $82(66.1)$ \\
\hline 61 & $2(1.6)$ & $4(3.1)$ & I $(0.8)$ \\
\hline 65 & 0 & $\mathrm{I}(0.8)$ & $3(2.4)$ \\
\hline 71 & 0 & $\mathrm{I}(0.8)$ & 0 \\
\hline Prior intravitreal anti-VEGF therapy, n (\%) & $8(6.3)$ & $7(5.5)$ & $8(6.5)$ \\
\hline $\mathrm{CRT}$, mean $(\mathrm{SD}) \mu \mathrm{m}$ & $526.3(164.4)$ & $520.3(154.8)$ & $527.7(170.5)$ \\
\hline Total NEI VFQ-25 score, mean (SD) & $67.9(17.1)$ & $68.9(16.9)$ & $69.7(17.1)$ \\
\hline Near activities subscale & $63.9(22.7)$ & $64.6(23.8)$ & $65.3(25.0)$ \\
\hline Distance activities subscale & $72.4(23.2)$ & $69.8(22.9)$ & $74.1(21.8)$ \\
\hline \multicolumn{4}{|l|}{ Chinese subgroup } \\
\hline & IVT-AFL 2q4 (N=10I) & IVT-AFL 2q8 (N=10I) & Laser photocoagulation ( $N=99)$ \\
\hline Female, n (\%) & $50(49.5)$ & $46(45.5)$ & $47(47.5)$ \\
\hline Mean age, years (SD) & $58.2(10.2)$ & $56.9(8.8)$ & $57.6(10.7)$ \\
\hline Mean $\mathrm{HbA}_{\mathrm{Ic}}, \%(\mathrm{SD})$ & $7.5(1.4)$ & $7.3(1.3)$ & $7.3(\mathrm{l} .4)$ \\
\hline Patients with $\mathrm{HbA}_{\mathrm{Ic}}>8 \%, \mathrm{n}(\%)$ & $30(29.7)$ & $29(28.7)$ & $26(26.3)$ \\
\hline Mean duration of diabetes, years (SD) & I2.I (7.3) & $10.0(7.3)$ & $11.6(6.8)$ \\
\hline Mean BCVA, letters (SD) & $54.1(12.4)$ & $57.2(12.2)$ & $54.4(14.5)$ \\
\hline Mean CRT, $\mu \mathrm{m}(\mathrm{SD})$ & $537.6(167.0)$ & $524.0(151.7)$ & $526.6(168.9)$ \\
\hline ETDRS DRSS score $\leq 53, \mathrm{n}(\%)$ & $98(97.0)$ & $96(95.0)$ & $95(96.0)$ \\
\hline
\end{tabular}

Notes: ${ }^{a} n=126,127$ and 124 , respectively. ${ }^{b} n=90,95$ and 79 , respectively.

Abbreviations: 2q4, 2 mg every 4 weeks; 2q8, 2 mg every 8 weeks; BCVA, best corrected visual acuity; CRT, central retinal thickness; DRSS, Diabetic Retinopathy Severity

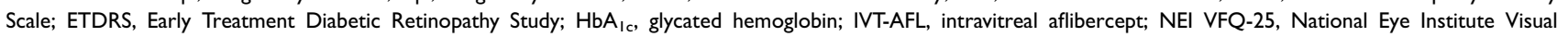
Functioning Questionnaire-25; SD, standard deviation; VEGF, vascular endothelial growth factor. 

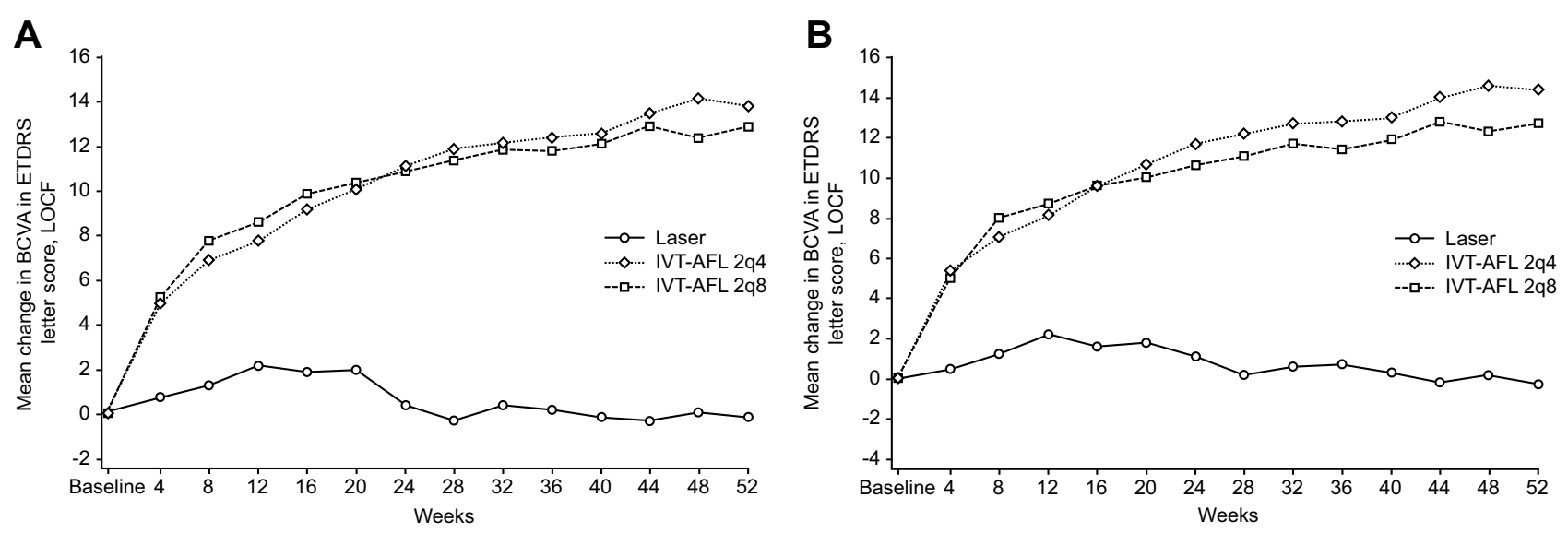

Figure 2 Change in BCVA from baseline to week 52 in the (A) Overall population and (B) Chinese subgroup.

Abbreviations: 2q4, 2 mg every 4 weeks; 2q8, 2 mg every 8 weeks; BCVA, best corrected visual acuity; ETDRS, Early Treatment Diabetic Retinopathy Study; IVT-AFL, intravitreal aflibercept; LOCF, last observation carried forward.

A

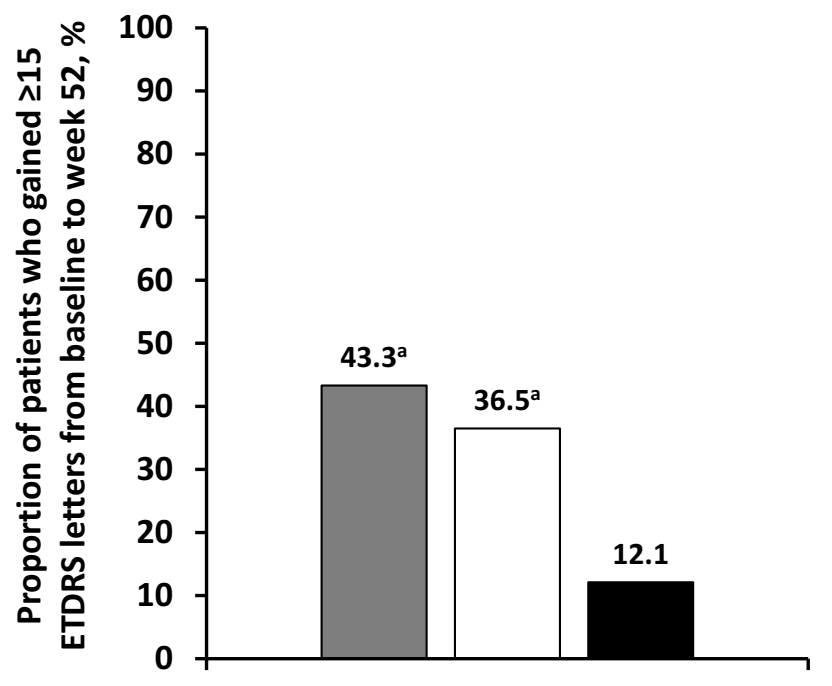

B

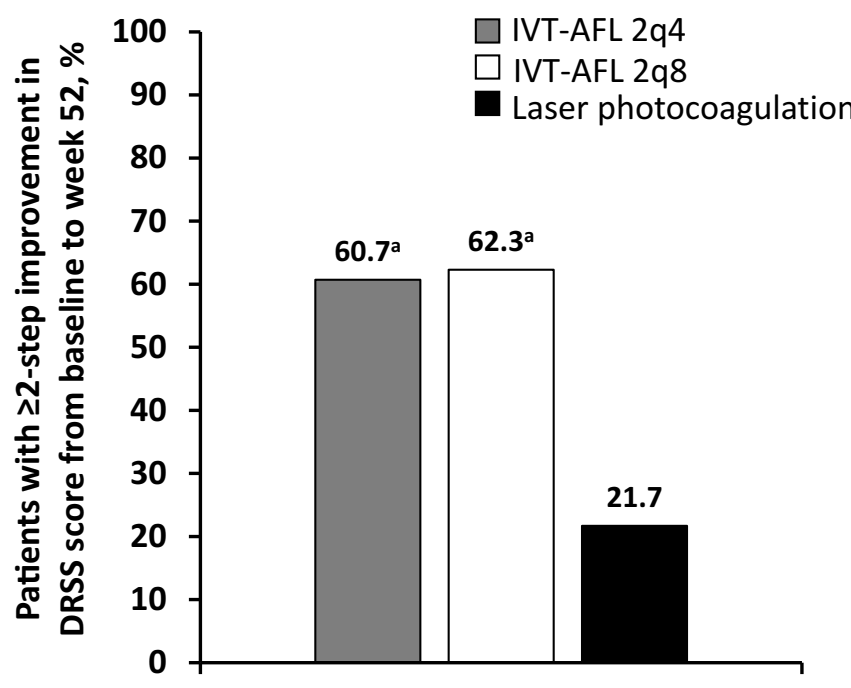

Figure 3 Proportion of patients gaining $\geq 15$ ETDRS letters (A) or a $\geq 2$-step improvement in DRSS (B) from baseline to week 52 . ${ }^{a} P<0.0001$. Abbreviations: 2q4, 2 mg every 4 weeks; 2q8, 2 mg every 8 weeks; DRSS, Diabetic Retinopathy Severity Scale; ETDRS, Early Treatment Diabetic Retinopathy Study; IVT-AFL, intravitreal aflibercept.

Visual and anatomic outcomes in the Chinese subgroup were consistent with the overall population. The mean (SE) change in BCVA from baseline to week 52 was $+14.1(0.7)$ and $+13.2(0.9)$ letters in the $2 \mathrm{q} 4$ and $2 \mathrm{q} 8$ groups, respectively, compared with -0.4 (3.4) in the laser photocoagulation group (both $\mathrm{P}<0.0001$ ) (Figure $2 \mathrm{~B}$ ). The mean change in CRT from baseline to week 52 was significantly greater in both IVT-AFL 2q4 and 2q8 groups compared with the laser group $(-245.0 \mu \mathrm{m}$ and $-244.9 \mu \mathrm{m}$, respectively, versus $-103.9 \mu \mathrm{m}$; both $\mathrm{P}<0.0001)$.

From baseline to week 52, the mean (SE) change in NEI VFQ-25 scores for near activities in the IVT-AFL $2 q 4$ and 2q8 groups was 5.30 (2.19) and 7.72 (2.03), respectively, compared with 1.04 (2.27) in the laser group $(\mathrm{P}<0.05$ for $2 \mathrm{q} 8$ versus laser treatment). For distance activities, the mean (SE) change from baseline to week 52 was 2.99 (2.00) and 8.62 (1.69) for the $2 \mathrm{q} 4$ and $2 \mathrm{q} 8$ groups, respectively, versus 1.95 (2.00) for the laser group ( $\mathrm{P}<0.05$ for the $2 \mathrm{q} 8$ group).

\section{Safety}

Incidence of ocular and nonocular treatment-emergent adverse events (TEAEs) was similar across groups (Table 2). No clinically meaningful ocular risk was observed in patients randomized to the IVT-AFL treatment groups compared with 
Table 2 Summary of Adverse Events by Treatment Group

\begin{tabular}{|c|c|c|c|}
\hline n (\%) & IVT-AFL 2q4 ( $N=127)$ & IVT-AFL 2q8 ( $N=\mid 27)$ & $\begin{array}{l}\text { Laser Photocoagulation } \\
(\mathrm{N}=124)\end{array}$ \\
\hline Any AE & $110(86.6)$ & II $3(89.0)$ & $112(90.3)$ \\
\hline \multicolumn{4}{|l|}{ Serious events } \\
\hline Any serious TEAE & $19(15.0)$ & $22(17.3)$ & $24(19.4)$ \\
\hline Any drug-related serious TEAE & 0 & I $(0.8)$ & $(0.8)^{\mathrm{a}}$ \\
\hline Any injection-related serious TEAE & 0 & 0 & $\mathrm{I}(0.8)^{\mathrm{a}}$ \\
\hline Any laser-related serious TEAE & 0 & 0 & I $(0.8)^{\mathrm{a}}$ \\
\hline \multicolumn{4}{|l|}{ Nonserious events } \\
\hline Any TEAE & $108(85.0)$ & $109(85.8)$ & III (89.5) \\
\hline Any drug-related TEAE & $4(3.1)$ & $7(5.5)$ & $4(3.2)$ \\
\hline Any injection-related TEAE & $19(15.0)$ & $23(18.1)$ & $8(6.5)$ \\
\hline Any laser-related TEAE & $3(2.4)$ & $3(2.4)$ & $3(2.4)$ \\
\hline $\begin{array}{l}\text { Any TEAEs leading to study drug } \\
\text { discontinuation }\end{array}$ & $4(3.1)$ & $4(3.1)$ & $4(3.2)$ \\
\hline Any ocular TEAE in the study eye & $65(51.2)$ & $60(47.2)$ & $72(58.1)$ \\
\hline \multicolumn{4}{|l|}{ Serious events } \\
\hline Any ocular serious $A E$ & $2(1.6)$ & $\mathrm{I}(0.8)$ & $6(4.8)$ \\
\hline Any drug-related ocular serious $\mathrm{AE}$ & 0 & 0 & $\mathrm{I}(0.8)$ \\
\hline Any injection-related ocular serious $A E$ & 0 & 0 & $\mathrm{I}(0.8)$ \\
\hline Any laser-related ocular serious $\mathrm{AE}$ & 0 & 0 & $\mathrm{I}(0.8)$ \\
\hline \multicolumn{4}{|l|}{ Nonserious events } \\
\hline Any drug-related ocular $\mathrm{AE}$ & $4(3.1)$ & $4(3.1)$ & $2(1.6)$ \\
\hline Any injection-related ocular $\mathrm{AE}$ & $19(15.0)$ & $23(18.1)$ & $8(6.5)$ \\
\hline Any laser-related ocular $A E$ & $3(2.4)$ & $3(2.4)$ & $3(2.4)$ \\
\hline Any nonocular TEAE & $79(62.2)$ & $81(63.8)$ & $77(62.1)$ \\
\hline \multicolumn{4}{|l|}{ APTC-classified events } \\
\hline Nonfatal myocardial infarction & $2(1.6)$ & $\mathrm{I}(0.8)$ & $\mathrm{I}(0.8)$ \\
\hline Nonfatal stroke & $2(1.6)$ & $3(2.4)$ & $\mathrm{I}(0.8)$ \\
\hline
\end{tabular}

Notes: ${ }^{a}$ One patient experienced three serious ocular events (glaucoma, hyphema, and vitreous hemorrhage) that were considered drug-related, injectionrelated, and laser-related.

Abbreviations: 2q4, 2 mg every 4 weeks; 2q8, 2 mg every 8 weeks; AE, adverse event; APTC, Antiplatelet Trialists' Collaboration; IVT-AFL, intravitreal aflibercept; TEAE, treatment-emergent adverse event.

the laser photocoagulation group. TEAEs were mild in intensity for most participants in the IVT-AFL groups. Moderate and severe ocular TEAEs were more common in the laser photocoagulation group.

In patients treated with IVT-AFL, the most frequently reported ocular TEAE in the study eye was conjunctival hemorrhage $(11.8 \%)$. All events of retinal hemorrhage $(8.7 \%)$, retinal aneurysm $(7.5 \%)$, and retinal exudates $(5.5 \%)$ were mild in intensity. Severe ocular TEAEs occurred infrequently in IVT-AFL-treated patients (eye pain, macular edema, ocular hypertension, posterior capsule opacification $[\mathrm{n}=1$ each]). Overall, incidences of increased intraocular pressure and cataract were $3.1 \%$ and $0.8 \%$ of patients, respectively, in both the IVT-AFL $2 q 4$ and $2 q 8$ treatment groups, with no observed differences between treatment groups. There were no reported cases of endophthalmitis or intraocular inflammation. One patient in the laser group experienced three serious ocular events (glaucoma, hyphema, and vitreous hemorrhage) that were considered drug-related, injection-related, and laser-related. The incidence of nonocular serious adverse events and arterial thromboembolic events defined by the Anti-Platelet Trialists' Collaboration criteria was low and similar across treatment groups $(3.1 \%, 2.4 \%$, and $1.6 \%$ of patients in the $2 \mathrm{q} 4,2 \mathrm{q} 8$, and laser groups, respectively). No deaths were reported in the study. 


\section{Discussion}

The VIVID-East study demonstrated the efficacy and safety of IVT-AFL for treating DME in Asian patients, including a large subgroup of Chinese patients, the largest subgroup represented in this study. Findings confirm that the treatment benefits of IVT-AFL are similar in Asian patients with DME from China, Russia, the Republic of Korea, and Hong Kong compared with the overall populations of the previously conducted VIVID and VISTA phase 3 studies. $^{6-8}$ Numerical improvement in BCVA was seen as early as week 4 (the first post-treatment time point) with IVT-AFL $2 q 4$ and 2q8. By week 20, BCVA had improved by a mean of 10.0 and 10.3 letters in the $2 \mathrm{q} 4$ and $2 \mathrm{q} 8$ groups, while the laser group had the largest improvement at week 12 (2.1 letters) and then slowly declined through to week 52 . The improvements in the IVT-AFL groups increased further through week 52. The numerical values for the mean change in BCVA at week 52 in the VIVID-East treatment groups were $+13.6(2 \mathrm{q} 4)$ and +13.1 (2q8) letters, compared with $+12.5(2 \mathrm{q} 4)$ and +10.7 letters $(2 \mathrm{q} 8)$ in VISTA,${ }^{6}+10.5(2 \mathrm{q} 4)$ and +10.7 letters in VIVID. ${ }^{6}$ The reasons for slightly different numerical outcomes are varied, and may include a younger mean age at study entry (58.5 years in the VIVIDEast study and 63.6 years in the VIVID study) or differences in mean baseline BCVA with 55.9 and 60.1 letters observed in the VIVID-East and VIVID studies, respectively. In addition, $>60 \%$ of Asian patients achieved a $\geq 2$-step improvement in DRSS score at week 52 . There was a robust and rapid reduction of CRT in both IVT-AFL groups, with significant improvements over the laser group. Again, this improvement was seen as early as week 4 .

It has previously been acknowledged that DME diagnosis and management remains suboptimal in many patients with diabetes, particularly in Asia. ${ }^{3}$ Reasons for this include limited screening, and many patients present late into the course of the condition with poor visual acuity. ${ }^{5}$ In China and Russia, more than $50 \%$ of patients with diabetes remain undiagnosed and untreated, ${ }^{1}$ which may ultimately result in irreversible vision loss. It has been highlighted in the literature that lack of access to eye specialists, lack of adequate clinical guidance and low awareness of diabetes, diabetic retinopathy, and DME limit the optimal management of these conditions in many Asian countries. ${ }^{5}$ As a consequence, healthcare centers and resources are affected by the increase in diabetes seen in Asia in recent decades. In addition, treatment choices are influenced by cultural factors and sometimes inadequate patient-physician interactions. Taken together, these challenges mean that patients in Asia tend to present with more advanced disease compared with other regions. The cost of anti-VEGF therapy is also a key barrier to treatment for patients in Asia with $\mathrm{DME}^{5}$ and, because the treatment of DME is not reimbursed in several Asian countries, the number of anti-VEGF injections currently administered by clinics is thought to be lower than in clinical trials. $^{5}$

Treatment recommendations for the management of Asian patients with DME are largely based on studies performed outside the region, with limited data generated from the Asian population. ${ }^{5}$ Recent evidence-based recommendations developed specifically for Asian countries highlight the need to enhance physician understanding of the importance of early intensive treatment to help optimize patient outcomes. ${ }^{5}$ While many Asian ophthalmologists still consider laser photocoagulation treatment as the mainstay treatment option, the latest recommendations suggest early and intensive anti-VEGF therapy as a first-line treatment for patients with center-involving DME with vision loss. In the present study, comparable outcomes observed in the IVT-AFL $2 \mathrm{q} 8$ versus $2 \mathrm{q} 4$ groups may indicate that initial therapy for the first 5 months followed by a reduced frequency of treatment is feasible without compromising efficacy. The latest recommendations for clinical management of DME in Asia state that the choice of anti-VEGF agent treatment depends on baseline visual acuity. ${ }^{5}$ Treatment guidelines highlight the Protocol T study, which demonstrated that in patients with poor baseline visual acuity who may present late, IVT-AFL may offer better results compared with alternative anti-VEGF treatment over 1 year. ${ }^{9}$ Furthermore, a previous study conducted in Asia demonstrated that IVT-AFL was an efficacious treatment option for DME in Asian patients who were refractory to monoclonal antibody-based anti-VEGF therapy. ${ }^{10}$ The safety outcomes from the current study were consistent with the known safety profile of IVT-AFL in DME. ${ }^{6-8}$ TEAEs were mild in intensity for most patients in the IVT-AFL groups. There were no cardiovascular events or deaths, which have previously been identified as potentially associated with anti-VEGF therapy in patients with DME. $^{5}$

While this study provides valuable information for the management of patients with DME in Asia, it must be acknowledged that physician and patient education regarding this condition, and the implementation of 
screening processes to identify patients earlier in their condition, are key to improving care. These factors would also enable earlier initiation of treatment, as advocated by treatment guidelines. Future, long-term studies providing real-world evidence regarding the management of patients with DME may provide further insights into how the challenges in the Asian region can be best addressed to ensure optimal treatment and reduced burden of care. This study offers important insights into management of the Asian population, with a focus on Chinese patients with DME, the largest population subgroup represented. It is recommended that future studies also include a focus on additional countries in the Asian region.

\section{Conclusions}

The 52-week VIVID-East study in Asian patients with DME demonstrated that IVT-AFL treatment every 4 or every 8 weeks (after 5 initial monthly doses) significantly improved visual outcomes and simultaneously improved the severity of diabetic retinopathy compared with laser photocoagulation. Treatment benefits were observed in the overall study population, including in Chinese patients, the largest subgroup included in the study. These significant visual and anatomic improvements mirrored those observed in the VISTA/VIVID phase 3 trials with IVT-AFL.

\section{Data Sharing Statement}

Availability of the data underlying this publication will be determined according to Bayer's commitment to the EFPIA/PhRMA "Principles for responsible clinical trial data sharing". This pertains to scope, time point, and process of data access.

As such, Bayer commits to sharing upon request from qualified scientific and medical researchers patient-level clinical trial data, study-level clinical trial data, and protocols from clinical trials in patients for medicines and indications approved in the United States (US) and European Union (EU) as necessary for conducting legitimate research. This applies to data on new medicines and indications that have been approved by the EU and US regulatory agencies on or after January 01, 2014.

Interested researchers can use www.clinicalstudydatare quest.com to request access to anonymized patient-level data and supporting documents from clinical studies to conduct further research that can help advance medical science or improve patient care. Information on the Bayer criteria for listing studies and other relevant information is provided in the Study sponsors section of the portal.

Data access will be granted to anonymized patient-level data, protocols, and clinical study reports after approval by an independent scientific review panel. Bayer is not involved in the decisions made by the independent review panel. Bayer will take all necessary measures to ensure that patient privacy is safeguarded.

\section{Acknowledgments}

The authors thank the patients and investigators who contributed to this study. Medical writing assistance for the preparation of this manuscript (under the guidance of the authors) was provided by Louise Brady, PhD (ApotheCom, UK). Medical writing and editorial assistance was funded by Bayer Consumer Care, AG, Pharmaceuticals, Switzerland. The VIVID-East study was sponsored by Bayer AG, Berlin, Germany. The sponsor participated in the design and conduct of the study, analysis of the data, and preparation of the manuscript.

\section{Disclosure}

YHY reports grants and/or personal fees from Allergan, Bayer, Alcon, and Roche, outside the submitted work. HW is an employee of Bayer HealthCare Inc, Pudong District, Shanghai, China. XR is an employee of Bayer HealthCare Inc, Chaoyang District, Beijing, China. FA was an employee of Bayer Pharmaceuticals, Berlin, Germany at the time this manuscript was written, he is now an employee of ProQR Therapeutics from Oct 01, 2019 onwards. The authors report no other conflicts of interest in this work.

\section{References}

1. International Diabetes Federation. IDF Diabetes Atlas. 9th; 2019. Available from: https://diabetesatlas.org/en/. Accessed May 5, 2019.

2. Boyer DS, Hopkins JJ, Sorof J, Ehrlich JS. Anti-vascular endothelial growth factor therapy for diabetic macular edema. Ther $A d v$ Endocrinol Metab. 2013;4(6):151-169. doi:10.1177/204201881351 2360

3. Chua J, Lim CXY, Wong TY, Sabanayagam C. Diabetic Retinopathy in the Asia-Pacific. Asia Pac J Ophthalmol. 2018;7(1):3-16. doi:10.22 608/APO.2017511

4. Stehouwer CDA. Microvascular dysfunction and hyperglycemia: a vicious cycle with widespread consequences. Diabetes. 2018;67 (9):1729-1741. doi:10.2337/dbi17-0044

5. Cheung GC, Yoon YH, Chen LJ, et al. Diabetic macular oedema: evidence-based treatment recommendations for Asian countries. Clin Exp Ophthalmol. 2018;46(1):75-86. doi:10.1111/ceo.2018.46.issue-1

6. Korobelnik JF, Do DV, Schmidt-Erfurth U, et al. Intravitreal aflibercept for diabetic macular edema. Ophthalmology. 2014;121 (11):2247-2254. doi:10.1016/j.ophtha.2014.05.006 
7. Brown DM, Schmidt-Erfurth U, Do DV, et al. Intravitreal aflibercept for diabetic macular edema: 100-week results from the VISTA and VIVID studies. Ophthalmology. 2015;122(10):2044-2052. doi:10.1016/j. ophtha.2015.06.017

8. Heier JS, Korobelnik JF, Brown DM, et al. Intravitreal aflibercept for diabetic macular edema: 148-week results from the VISTA and VIVID studies. Ophthalmology. 2016;123(11):2376-2385. doi:10.1016/j. ophtha.2016.07.032
9. Wells JA, Glassman AR, Ayala AR, et al. Aflibercept, bevacizumab, or ranibizumab for diabetic macular edema. $N$ Engl J Med. 2015;372 (13):1193-1203.

10. Chen YY, Chang PY, Wang JK. Intravitreal aflibercept for patients with diabetic macular edema refractory to bevacizumab or ranibizumab: analysis of response to aflibercept. Asia Pac J Ophthalmol. 2017;6(3):250-255.

\section{Publish your work in this journal}

Clinical Ophthalmology is an international, peer-reviewed journal covering all subspecialties within ophthalmology. Key topics include: Optometry; Visual science; Pharmacology and drug therapy in eye diseases; Basic Sciences; Primary and Secondary eye care; Patient Safety and Quality of Care Improvements. This journal is indexed on PubMed

Submit your manuscript here: https://www.dovepress.com/clinical-ophthalmology-journal
Central and CAS, and is the official journal of The Society of Clinical Ophthalmology (SCO). The manuscript management system is completely online and includes a very quick and fair peer-review system, which is all easy to use. Visit http://www.dovepress.com/ testimonials.php to read real quotes from published authors. 